\title{
DIRECTED SEARCH OF VARIANTS IN TECHNOLOGIES FOR REENGINEERING OF CORPORATE COMPUTER NETWORKS
}

\author{
Beskorovainyi V., Russkin V.
}

The formalization of the multicriteria task of system reengineering of a corporate computer network is proposed. It presupposes structural, topological, parametric and technological optimization of the network in terms of a complex "effect-cost" indicator. The parameters of the quality of the variants are detailed in the form of particular criteria of costs, efficiency, reliability and survivability. This allows us to reduce the multicriteria task to a traditional optimization task with a scalar criterion. The proposed approach to the formation of a subset of effective ones already at the stage of generation of feasible variants can significantly reduce the time and capacitive complexity of methods for solving the task of network reengineering. For the final choice of the reengineering variant by the decision maker, it is proposed to reduce to the required size the subsets of effective alternatives using the known combined method.

\section{Introduction}

The effectiveness of corporate computer networks (CCN) is largely determined by the way they are structured. Variants of network structures can be implemented on different sets of elements, different architectures and channels for organizing connections between elements and nodes [1]. Changes in the requirements for the functional characteristics of existing CCNs, improvement of technologies and information processing facilities at a certain stage lead to the need for their modernization. Fundamental changes in the structure, topology, parameters and technology of networks functioning are implemented in the process of their reengineering [2].

The most important features of CCN, which are taken into account in their reengineering projects, include [3]: close interconnection of structural, topological, parametric and technological optimization tasks and the complexity of their joint solution; the combinatorial nature of the most tasks of the 
problem and the lack of effective methods for their solution; incomplete certainty of the initial data and purposes of creation; the complexity of functioning technologies does not allow the use of analytical methods for their analysis.

The process of reengineering involves many specific tasks of system design of CCN [4-5]: Task $_{1}$ - selection of principles of CCN construction; Task ${ }_{2}$ - selection of network structure; Task $_{3}$ - determination of topology of elements and links; Task - selection of functioning technology; Task $_{5}$ - determination of parameters of elements and links; Task $_{6}$ - evaluation of efficiency of permissible network design variants $s \in S$ and selection of the best of them $s^{o} \in S$ (where $S$ - many permissible variants of network reengineering).

When solving CCN optimization tasks as large-scale objects, an approach is used that involves sequentially decomposing their goals, functions and tasks and then aggregating them at the appropriate levels to generate variants of their construction as a whole.

Most CCN system design tasks belong to the class of combinatorial, accurate solutions of which has non-polynomial temporal complexity [4]. This creates computational tasks in many practical tasks. In view of this, the scientific and applied task of improving the efficiency of CCN reengineering technologies by developing effective methods of system optimization, using procedures for allocating effective solutions and targeting variants.

\section{BASIC STATEMENT OF THE REENGINEERING TASK}

In the early stages of reengineering, the task of optimizing the CCN construction variant is formalized in terms of "condition-target." The conditions define the initial data and limitations of the task, and the goal is the best version of building a network from a set of permissible $s^{o} \in S$, according to a set of specific 
efficiency criteria $K(s)=\left\{k_{1}(s), k_{2}(s), \ldots, k_{m}(s)\right\} \quad$ [6]. The functional effect of using CCN is generally a non-decreasing function of the $r$ esources (cost) $\bar{Q}(s)=F[\bar{C}(s)]$ spent to achieve it (where $\bar{Q}(s)$ and $\bar{C}(s)$ are generalized scalar estimates of the effect and cost of the network variant; $F$ is an operator that displays a resource utilization strategy).

Under conditions of specified restrictions on effect indicators $\bar{Q}(s) \geq \bar{Q}^{*}$ and (or) costs $\bar{C}(s) \leq \bar{C}^{*}$, the task of network reengineering can be presented in the form:

$$
s^{o}=\arg \max _{s \in S}\left[\bar{Q}(s) / \bar{C}(s): \bar{Q}(s) \geq \bar{Q}^{*}, \bar{C}(s) \leq \bar{C}^{*}\right]
$$

where $\bar{Q}^{*}, \bar{C}^{*}$ are marginal levels of the generalized estimates of the effect and costs of CCN reengineering.

Specific cases of task (1) are the tasks of selecting an variant that maximizes the given effect under conditions of given resource restrictions $\bar{C}(s) \leq \bar{C}^{*}$ or an variant that minimizes the given costs to obtain a given level of effect $\bar{Q}(s) \geq \bar{Q}^{*}$.

The most essential and general requirements for CCN are efficiency, reliability, economy and survivability.

The basic CCN reengineering task is considered in the following setting. The existing version of the network construction $s^{\prime} \in S$, as well as the set of: users $I=\{i\}, i=\overline{1, n}_{O}$, and their characteristics (territorial location, the need to perform information and computational work); typical nodes $\Omega_{U}=\{\omega\}$ and links $\Omega_{C}=\{\omega\}$ , on the basis of which the network is synthesized; places of possible placement of its nodes $G=\{g\}$; acceptable technologies for its functioning $\Gamma=\{\gamma\}$.

It is necessary to determine the CCN reengineering variant $s \in S$ : the number of network nodes $n_{U}$; their types $X=\left\{x_{i}^{\omega}\right\}, i=\overline{1, n_{U}}, \omega \in \Omega_{U}$; location of nodes 
$Y=\left\{y_{i}^{g}\right\}, \quad i=\overline{1, n_{U}}, \quad g \in G ; \quad$ set and types of links between elements and nodes $R=\left\{r_{i j}^{\omega}\right\}, \quad i, j=\overline{1, n_{o}}, \omega \in \Omega_{C}$; subsets of users associated with each of the nodes and the technology of their functioning $Z=\left\{z_{i}^{\gamma}\right\}$, $i=\overline{1, n_{U}}, \gamma \in \Gamma$. At the same time, the desired goal is to extremize the set of particular performance criteria for the selected CCN reengineering variant $s^{o} \in S$ :

- costs

$$
k_{1}(s)=C\left(n_{U}, X, Y, R, Z\right) \rightarrow \min _{n_{U}, X, Y, R, Z}
$$

- efficiency (access time)

$$
k_{2}(s)=\tau\left(n_{U}, X, Y, R, Z\right) \rightarrow \min _{n_{U}, X, Y, R, Z} ;
$$

- reliability

$$
k_{3}(s)=\psi\left(n_{U}, X, Y, R, Z\right) \rightarrow \max _{n_{U}, X, Y, R, Z}
$$

- survivability

$$
k_{4}(s)=\mu\left(n_{U}, X, Y, R, Z\right) \rightarrow \max _{n_{U}, X, Y, R, Z} .
$$

Reengineering costs (2) include the costs of creating new nodes and connections between users and nodes. The access time to network resources (3) includes the time spent at all stages of the process, including the waiting times, processing and transmission of requests and responses between elements and nodes. To evaluate it, it is proposed to use its average, weighted average or maximum value for all network users. As an estimate of the reliability (4), it is proposed to use the probability of the network being in a working state. For the indicator (5), it is proposed to use the estimates of the structural and functional survivability of the network, obtained on the basis of the 
analysis of its topological structure, as well as the reliability of its elements and connections.

Detailed the indicators in the form of particular criteria (2) - (5) allows us to reduce the multicriteria $\mathrm{CCN}$ reengineering task to the traditional optimization task with a scalar criterion of overall utility [7-9]:

$$
\begin{gathered}
P(s)=\sum_{i=1}^{4} \eta_{i} \xi_{i}(s), \\
\xi_{i}(s)=\xi_{i}\left(k_{i}(s)\right)=\left(\frac{k_{i}(s)-k_{i}^{-}(s)}{k_{i}^{+}(s)-k_{i}^{-}(s)}\right)^{\gamma_{i}}, i=\overline{1,4}
\end{gathered}
$$

where $\eta_{i}$ is a weighting factor taking into account the importance of a particular criterion $k_{i}(s), \eta_{i} \geq 0, \sum_{i=1}^{4} \eta_{i}=1 ; \xi_{i}(s)=\xi\left(k_{i}(s)\right)$ is a particular criterion utility function $k_{i}(s) ; k_{i}(s)$ - value of a particular criterion; $k_{i}^{-}(s), k_{i}^{+}(s)-$ respectively, the worst and the best value of the particular criterion; $\gamma_{i}-$ parameter that determines the type of dependence (7): at $\gamma_{i}=1$ - linear; at $0<\gamma_{i}<1-$ convex up; $\gamma_{i}>1-$ convex down.

Regardless of the method for solving the task, it is advisable to preliminary select a subset of reengineering variants that are effective $S^{E} \subset S$ by criteria $k_{i}(s)$, $i=\overline{1,4}$.

\section{FORMATION OF SUBSETS OF EFFECTIVE REENGINEERING VARIANTS}

The set of valid CCN reengineering variants consists of disjoint subsets of effective $S^{E}$ and ineffective $S^{N}$ variants:

$$
S=S^{N} \cup S^{E}, S^{N} \cap S^{E}=\varnothing,\left|S^{N}\right|>>\left|S^{E}\right| .
$$


A CCN reengineering variant $s \in S^{E}$ will be called effective (Pareto-optimal, non-dominant) if on the set of feasible variants $S$ for the selected particular criteria $k_{i}(s), i=\overline{1,4}(2)$-(5) there is no variant $s \in S$ for which the inequalities are satisfied [9]:

$$
\begin{gathered}
k_{i}(s) \geq k_{i}\left(s^{E}\right) \text {, если } k_{i}(s) \rightarrow \max , \\
k_{i}(s) \leq k_{i}\left(s^{E}\right) \text {, если } k_{i}(s) \rightarrow \min
\end{gathered}
$$

and at least one of them was strict.

In the tasks of multi-criteria choice, the set of admissible variants $S=\{s\}$ is considered to be given. Depending on the dimension of the task $|S|$, various methods and algorithms are used to select a subset of effective variants: discrete choice, weighting method, pairwise comparisons, methods based on Karlin and Hermeyer 's theorems, evolutionary search based on genetic algorithms [10-12].

The subset of effective solutions $S^{E}$ based on Karlin's theorem is determined by combining variants $s_{i}^{o}, i=\overline{1, m}$, that optimize each of the local criteria $k_{i}(s)$ with solutions to the parametric programming task [13]:

$$
\begin{aligned}
& \lambda_{i} \in \Lambda=\left\{\lambda_{i}: \lambda_{i}>0 \quad \forall i=\overline{1, m}, \sum_{i=1}^{m} \lambda_{i}=1\right\}, \\
& s_{i}^{o}=\arg \max _{s \in S}\left\{P(s)=\sum_{i=1}^{m} \lambda_{i} \xi_{i}(s)\right\} .
\end{aligned}
$$

The subset of effective variants based on Hermeier's theorem is determined by combining variants $s_{i}^{o}, i=\overline{1, m}$ that optimize each of the local criteria $k_{i}(s)$ with solutions to the parametric programming task [13]:

$$
\begin{aligned}
& \lambda_{i} \in \Lambda=\left\{\lambda_{i}: \lambda_{i}>0 \quad \forall i=\overline{1, m}, \sum_{i=1}^{m} \lambda_{i}=1\right\}, \\
& s_{i}^{o}=\arg \max _{s \in S}\left\{P(s)=\min _{i} \lambda_{i} \xi_{i}(s)\right\} .
\end{aligned}
$$

In this case: the methods of discrete choice and pairwise comparisons, due to 
the high time complexity, are applicable only on relatively small sets of feasible solutions $S=\{s\}$; evolutionary, weighted methods, including methods based on Karlin and Hermeyer theorems, have a lower adjustable time complexity, but in the general case, they allow to single out only incomplete subsets $S^{E}$ (Pareto fronts) [14].

To reduce the time and capacitive complexity of methods for solving the CCN reengineering task, it is proposed to form a subset of effective $S^{E}$ already at the stage of generation of feasible variants $S$.

\section{CHOOSING THE BEST VARIANT BY THE DIRECTED SEARCH METHOD}

The analysis of the dependences of the envelopes of local extremes of the functions of partial cost criteria $k_{1}(s)$ (2), efficiency $k_{2}(s)$ (3), reliability $k_{3}(s)$ (4) and survivability $k_{4}(s)$ (5) on the number of network nodes $n_{U}$ made it possible to reveal such features [16]:

- functions (3) - (5) have the best values at $n_{U}=1$;

- on the segment $1 \leq n_{U} \leq n_{U \text { max }}$ (where $n_{U \text { max }}$ is the maximum effective number of network nodes), the utility functions of the particular criteria (7) indicators of efficiency $\xi_{2}\left(s, n_{U}\right)$ and survivability $\xi_{4}\left(s, n_{U}\right)$ are one-extreme;

- the utility function of a particular criterion of reliability indicators $\xi_{3}\left(s, n_{U}\right)$ is monotonically decreasing;

- the envelope of local extremes, the utility function of the private cost criterion $\xi_{1}\left(s, n_{U}\right)$ can be multi-extremal.

To solve the task of forming a subset of effective CCN reengineering variants, this requires the use of methods that allow taking into account the possible multiextremality of the general utility function $P\left(s, n_{U}\right)(6)$ on a segment. The utility functions of particular criteria (7) for the indicators of efficiency (3), reliability (4) 
and survivability (5) decrease in the initial segment $1 \leq n_{U} \leq n_{U \text { max }}$. In order for at least one solution to task (1) to exist, it is necessary that its constraints are satisfied at least for. With this in mind, to solve the task, it is proposed to use modifications of the directed search method with the addition of a procedure for calculating the value of the additive convolution of partial criteria $P\left(s, n_{U}\right)(6)[16]$.

The proposed procedure for forming a subset of effective CCN reengineering variants $S^{E}$ includes the following sequence of steps.

1. Setting the initial values of the number of nodes $n_{U}=1$, variant number $i:=0$ set of effective variants $S^{E}=\varnothing$.

2. Transition to a new variant $i:=i+1$. Generate a variant $s_{i} \in S$ and calculate estimates of cost indicators $k_{1}\left(s_{i}\right)$ (2), efficiency $k_{2}\left(s_{i}\right)$ (3), reliability $k_{3}\left(s_{i}\right)$ (4), survivability $k_{4}\left(s_{i}\right)(5)$.

3. Compare an variant $s_{i}$ with each variant $s_{j} \in S^{E} j=\overline{1,\left|S^{E}\right|}$. If at least one variant from $S^{E}$ dominates on $s_{j}$, exclude from further consideration and go to step 4. Otherwise, add $s_{i}$ to the subset of effective $S^{E}$ and remove from the subset $S^{E}$ all $s_{j}$ that are worse than $s_{i}$ for all indicators (2) - (5).

4. If the termination conditions for the used method of forming the set of feasible reengineering variants $S$ are not met, go to step 2; otherwise, go to step $5 .$.

5. If the best values of all indicators (2) - (5) for the value $n_{U}$ are worse than for $n_{U}-1$, go to step 6 ; otherwise $n_{U}:=n_{U}+1$, $i:=0$, and go to step 2.

6. Completion of the procedure: highlighted a subset of effective CCN reengineering variants $S^{E}$.

For the final choice of the CCN reengineering variant by the decision maker, it is advisable to reduce the subset of alternatives $S^{E}$ to the required size using the well-known combined method [16]. For the final selection of the best CCN 
reengineering variant from the subset of effective ones, the well-known multicriteria selection methods can be used [7-9].

\section{CONCLUSIONS}

The formalization of the multicriteria task of reengineering of a corporate computer network is carried out, which assumes its structural, topological, parametric and technological optimization according to the complex indicator "effect-costs". Detailing the quality indicators of variants in the form of particular criteria of costs, efficiency, reliability and survivability allows us to reduce it to a traditional optimization task with a scalar criterion. The proposed approach to the formation of a subset of effective ones already at the stage of generation of feasible variants can significantly reduce the time and capacitive complexity of methods for solving the task of network reengineering. For the final choice of the reengineering variant by the decision maker, it is advisable to reduce the subset of effective alternatives to the required size using a known combined method.

The direction of further research can be the development of effective methods of directed enumeration of reengineering variants for situations of incomplete determination of goals and data.

\section{REFERENCES}

1. Tannenbaum, E.S., Ueseroll, D. (2018), Computer networks, SPb., Piter, 960 p. (in Russian).

2. Nesterenko, S.F. (2017), "Choosing the optimal plan for energy efficient reengineering of a corporate computer network", Electrical and computer systems, No. 25, P. 341-346 (in Russian).

3. Beskorovainyi, V.V. (2002), "Systemological analysis of the problem of structural synthesis of geographically distributed systems", Автоматизированные системь управления и приборы автоматики, Vol. 120, P. 29-37 (in Russian).

4. Beskorovainyi, V.V., Podoliaka, K.E. (2015), "Development of systemological model of the problem of structural and topological reengineering of large-scale monitoring system", Eastern-European Journal of Eenterprise Technologies, No. 3(75), P. 37-42 (in Russian). 
5. Beskorovainyi, V.V., Podoliaka, K.E. (2015), "Development of a model for a multicriteria task of reengineering the topological structures of large-scale monitoring systems", Eastern-European Journal of Eenterprise Technologies, No. 4(76), P. 49-55.

6. Beskorovainyi, V., Imanhulova, Z. (2017), "Technology of large-scale objects system optimization", ECONTECHMOD, Vol. 06, No. 4, P. 3-8.

7. Kruchkovskyi, V.V., Petrov, E.G., Sokolova, N.A., Hodakov, V.E. (2013), Introduction to the normative theory of decision-making, Herson, Grin D.S., 284 p. (in Russian).

8. Petrov, E, Brynza, N, Kolesnyk, L, Pisklakova, O. (2014), Methods and models of decisionmaking under conditions of multi-criteria and uncertainty, Herson, Grin D.S., 192 p.

9. Ovezgeldiev, A.O., Petrov, E.G., Petrov, K.E. (2002), Synthesis and identification of multifactorial models for estimation and optimization, Kiev: Naukova dumka, 164 p.

10. Bezruk, V.M., Chebotareva, D.V., Skorik, Ju.V. (2017). Multi criteria analysis and selection of telecommunication means, Kharkov, FOP Korjak S.F., 268 p.

11. Chebotareva, D.V., Bezruk, V.M. (2013). Multi criteria optimization of design solutions when planning cellular networks of mobile communications, Kharkov, Company SMIT, 148 p. (in Russian).

12. Kalyanmoy Deb and Debayan Deb. (2014), "Analysing mutation schemes for real-parameter genetic algorithms", International Journal of Artificial Intelligence and Soft Computing, No. 4(1), P. 1-28.

13. Michalevich, V.S., Volrovich, V.L. (1982), Computational methods of research and design of complex systems, M., Nauka, 288 p. (in Russian).

14. Vladimir V. Beskorovainyi, Lubomyr B. Petryshyn, Olha Yu. Shevchenko (2020), "Specific subset effective option in technology design decisions", Applied Aspects of Information Technology, Vol.3, No.1, P. 443-455.

15. Beskorovainyi, V.V., Podoliaka, K.E. (2016), "Choice of multi criteria solutions in the reengineering of the topological structures of large-scale monitoring systems", Information processing systems, No. 5(142), P. 80-86 (in Russian).

16. Beskorovainyi, V.V., Podoliaka, K.E. (2015), "Modifications of the directed search method for reengineering the topological structures of large-scale monitoring systems", Radioelectronics and Informatics, No. 3 (70), P. 55-62 (in Russian).

17. Beskorovainyi, V. (2020), "Combined method of ranking options in project decision support systems", Innovative Technologies and Scientific Solutions for Industries, No 4 (14), P. 13-20. DOI: https://doi.org/10.30837/ITSSI.2020.14.013. 